\title{
Descending Flight Simulation of Tiltrotor Aircraft at Different Descent Rates
}

\author{
Ayato Takii ${ }^{1(\bowtie)}$, Masashi Yamakawa ${ }^{1}$, and Shinichi Asao ${ }^{2}$ \\ ${ }^{1}$ Kyoto Institute of Technology, Matsugasaki, \\ Sakyo-ku, Kyoto 606-8585, Japan \\ kpp_fsl_ta@yahoo.co.jp \\ 2 College of Industrial Technology, 1-27-1, Amagasaki, Hyogo 661-0047, Japan
}

\begin{abstract}
Helicopters and tiltrotor aircrafts are known to fall into an unstable state called vortex ring state when they descend rapidly. This paper presents a six degrees of freedom descending flight simulation of a tiltrotor aircraft represented by the V-22 Osprey, considering the interaction between fluid and a rigid body. That is, an aircraft affects the surrounding flow field by rotating the rotors, and flies with the generated force as thrust. Similarly, an orientation of the airframe is controlled by aerodynamic force which is generated by manipulating the shape. This numerical analysis is a complicated moving boundary problem involving motion of an air-frame or rotation of rotors. As a numerical approach, the Moving Computational Domain (MCD) method in combination with the multi-axis sliding mesh approach is adopted. In the MCD method, the whole computational domain moves with objects in the domain. At this time, fluid flow around the objects is generated by the movement of the boundaries. In addition, this method removes computational space restrictions, allowing an aircraft to move freely within the computational space regardless of a size of a computational grid. The multi-axis sliding mesh approach allows rotating bodies to be placed in a computational grid. Using the above approach, the flight simulation at two different descent rates is performed to reveal a behavior of a tiltrotor aircraft and a state of the surrounding flow field.
\end{abstract}

Keywords: Computational fluid dynamics - Tiltrotor - Flight simulation · Vortex ring state

\section{Introduction}

It is known that rotorcrafts such as a helicopter or a tiltrotor fell into unstable state called vortex ring state (VRS) when it descends at high descent rate or at an angle close to vertical descent. At this time, lift generated by the rotor disc is significantly reduced. This phenomenon occurs when the descent speed approaches the rotor wake speed and airflow recirculate through the rotor disk. VRS has also caused several rotorcraft crashes. VRS accidents have also been reported on a tiltrotor V-22 Osprey which has recently attracted attention. This was due to the pilot descending beyond the recommended descent range [1]. The behavior of rotors in VRS has long been known to aerodynamic experts, and a considerable number of studies have been reported [2]. Many researches have been investigating the VRS characteristics of the rotor by 
experimental tests and numerical simulations [3, 4]. However, most of these studies focused on flight tests or wind tunnel tests in steady conditions. Vortex ring state is a complex phenomenon with large unsteady airflow. Hence practical investigations can also be useful to fully understand aerodynamics and develop accurate prediction methods. Researches on unsteady simulation of helicopters have been conducted [5, 6]. However, few studies on tiltrotor aircraft have been seen.

The purpose of this study is to compute unsteady flow around a tiltrotor aircraft when it descends and to simulate the motion of the aircraft in such airflow. The six degrees of freedom descending flight simulation is performed on a tiltrotor aircraft represented by V-22 Osprey, considering an interaction between fluid and a rigid body. By performing numerical computations at different descent rates, effects on motion of an aircraft and complex fluid phenomena caused by aircraft movement are analyzed. To perform descent flight simulations, coupled computation is conducted that integrate both flight dynamics which deals with aircraft movement and fluid dynamics which deals with fluid flow around aircraft. In other words, the aircraft is treated as a rigid body and flies under force generated by interaction with the surrounding fluid flow. To solve such a complicated moving boundary problem, a combination of the MCD method based on the unstructured moving-grid finite-volume method and the multi-axis sliding mesh method was adopted as numerical approach.

\section{Numerical Approach}

\subsection{Governing Equation}

To solve the flow field around a tiltrotor aircraft, three-dimensional Euler equations are used as governing equations. The equations in the conservation form are written as follows:

$$
\begin{gathered}
\frac{\partial \boldsymbol{q}}{\partial t}+\frac{\partial \boldsymbol{E}}{\partial x}+\frac{\partial \boldsymbol{F}}{\partial y}+\frac{\partial \boldsymbol{G}}{\partial z}=0 \\
\boldsymbol{q}=\left[\begin{array}{c}
\rho \\
\rho u \\
\rho v \\
\rho w \\
e
\end{array}\right], \boldsymbol{E}=\left[\begin{array}{c}
\rho u \\
\rho u^{2}+p \\
\rho u v \\
\rho u w \\
u(e+p)
\end{array}\right], \boldsymbol{F}=\left[\begin{array}{c}
\rho v \\
\rho u v \\
\rho v^{2}+p \\
\rho v w \\
v(e+p)
\end{array}\right], \boldsymbol{G}=\left[\begin{array}{c}
\rho w \\
\rho u w \\
\rho v w \\
\rho w^{2}+p \\
w(e+p)
\end{array}\right]
\end{gathered}
$$

where $\boldsymbol{q}$ is the conserved quantity vector, $\boldsymbol{E}, \boldsymbol{F}, \boldsymbol{G}$ are the inviscid flux vectors. As unknowns, $\rho$ is the density, $u, v, w$ are the $x, y, z$ components of the velocity vector and $e$ is the total energy per unit volume. The working fluid assumed to be perfect gas, the pressure $p$ is defined as follows:

$$
p=(\gamma-1)\left[e-\frac{1}{2} \rho\left(u^{2}+v^{2}+w^{2}\right)\right],
$$


where $\gamma$ is the specific heat ratio. In this paper, $\gamma$ is taken as 1.4 , and the initial conditions of density, pressure, velocity components in the $x, y$ and $z$ directions are given by $\rho=1.0, p=1.0 / \gamma, u=0.0, v=0.0, w=0.0$, respectively.

\subsection{Unstructured Moving-Grid Finite-Volume Method}

The unstructured moving-grid finite-volume method [7] is used to perform computation involving movement and deformation of grids. In the method, fluxes are evaluated on a control volume in the space-time unified domain $(\mathrm{x}, \mathrm{y}, \mathrm{z}, \mathrm{t})$ so that the geometric conservation law (GCL) [8] is satisfied. By applying the divergence theorem to spacetime unified control volume, the three-dimensional Euler equations are deformed as follows:

$$
\begin{aligned}
\int_{\Omega}\left(\frac{\partial \boldsymbol{q}}{\partial t}+\frac{\partial \boldsymbol{E}}{\partial x}+\frac{\partial \boldsymbol{F}}{\partial y}+\frac{\partial \boldsymbol{G}}{\partial z}\right) d \Omega & =\int_{\partial \Omega}(\boldsymbol{E}, \boldsymbol{F}, \boldsymbol{G}, \boldsymbol{q}) \cdot \tilde{\boldsymbol{n}} d \mathrm{~V} \\
& =\sum_{l=1}^{6}\left(\boldsymbol{E} \tilde{n}_{x}+\boldsymbol{F} \tilde{n}_{y}+\boldsymbol{G} \tilde{n}_{z}+\boldsymbol{q} \tilde{n}_{t}\right)_{l}=0
\end{aligned}
$$

where $\Omega$ is the control volume, $\tilde{\boldsymbol{n}}=\left(\tilde{n}_{x}, \tilde{n}_{y}, \tilde{n}_{z}, \tilde{n}_{t}\right)$ is the outward unit normal vector on $\partial \Omega$ and $l$ indicates faces of the control volume.

\subsection{Moving Computational Domain Method}

In this paper, the flow field around a tiltrotor aircraft that occurs when the aircraft descends is computed. In the traditional approach, an aircraft is placed in a uniform flow and a flow around that is computed. However, it cannot be applied to this simulation involving free movement of an aircraft. Such an analysis needs to be treated as a moving boundary problem. The moving computational domain (MCD) method [9-14] is a technique for computing fluid flow around moving object. In this method, whole computational domain moves with an object which is inside the domain as shown in Fig. 1. At this time, fluid flow around an object is generated by movement of boundary surfaces. Specifically, it is caused by the boundary conditions given to the moving boundary. In addition, this method removes computational space limitations. Therefore, an aircraft can move freely in the calculation space regardless of the size of computational grid. Combining with the multi-axis sliding mesh approach [15], this method is applied not only to flight of an aircraft, but also to rotation of two rotors. The computational procedure is based on the unstructured moving-grid finite-volume method described in the last subsection. Flow variables are defined at the center of cells in unstructured mesh. The flux vectors are evaluated using the Roe flux difference splitting scheme [16] with MUSCL scheme. Gradient limiter is Hishida's limiter of van Leer type [17]. The two-stage Runge-Kutta method is used to solve local time stepping. 


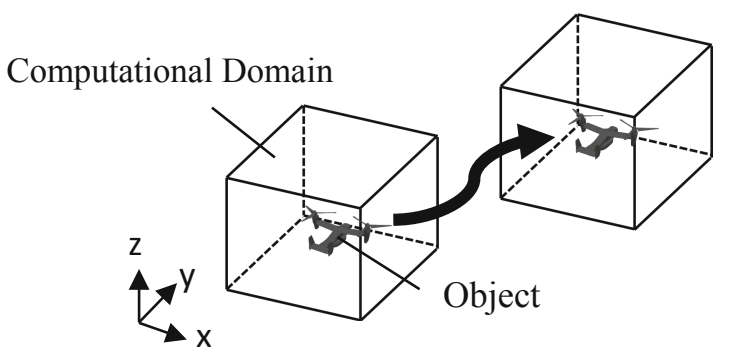

Fig. 1. Conceptual figure of the MCD method

\subsection{Coupled Computation}

In this paper, position and orientation of a tiltrotor aircraft is determined by coupled computation by considering the interaction between the body and surrounding fluid flow. An aircraft is considered as rigid body in three-dimensional space. Therefore, the motion of an aircraft is determined by solving the equations of motion of six degrees of freedom, consisting of three degrees of freedom translation and rotation. Newton's law of motion and Euler's rotation equation are used for translation and rotation, respectively. Each equation of motion is as follows:

$$
\begin{gathered}
m \frac{d^{2} \boldsymbol{r}}{d t^{2}}=\boldsymbol{F}, \\
I \frac{d \omega}{d t}+\omega \times I \omega=\boldsymbol{T},
\end{gathered}
$$

where $m$ is the mass of the aircraft, $\boldsymbol{r}$ is the position vector of the center of the aircraft, $\boldsymbol{F}$ is the force vector, $I$ is the inertia tensor (written in matrix form), $\omega$ is the angular velocity vector and $\boldsymbol{T}$ is the torque vector around the center. The torque and force are calculated by integrating the pressure on surface of the aircraft. These equations are discretized as follows:

$$
\begin{gathered}
m \boldsymbol{v}^{n+1}=m \boldsymbol{v}^{n}+\boldsymbol{F}^{n} \Delta t \\
\boldsymbol{r}^{n+1}=\boldsymbol{r}^{n}+0.5\left(\boldsymbol{v}^{n+1}+\boldsymbol{v}^{n}\right) \Delta t \\
I \boldsymbol{\omega}^{n+1}=I \boldsymbol{\omega}^{n}+\left(\boldsymbol{T}^{n}-\boldsymbol{\omega}^{n} \times I \boldsymbol{\omega}^{n}\right) \Delta t,
\end{gathered}
$$

where a subscript $n$ is the time level and $\Delta t$ is the time step. 


\section{Flight Simulation of Tiltrotor Aircraft}

\subsection{Computational Mesh}

$\mathrm{V}-22$ Osprey which is the major tilt-rotor aircraft was employed as a computational model. The overall length is $17.48 \mathrm{~m}$, the weight is $21545 \mathrm{~kg}$ and the rotor speed is $397 \mathrm{rpm}$. The overall length $L$ is the representative length. A computational domain and surface grid of a tiltrotor aircraft is shown in Fig. 2. The total number of cells is 3297890 and the size of computational $30 \mathrm{~L}$. This grid was created by using MEGG3D $[18,19]$. In this computation, the computational domain is divided into 7 domains to use multi-axis sliding mesh approach. Figure 3 shows domain decomposition. Computational grids for each domain are shown from Figs. 4, 5, 6 and 7.

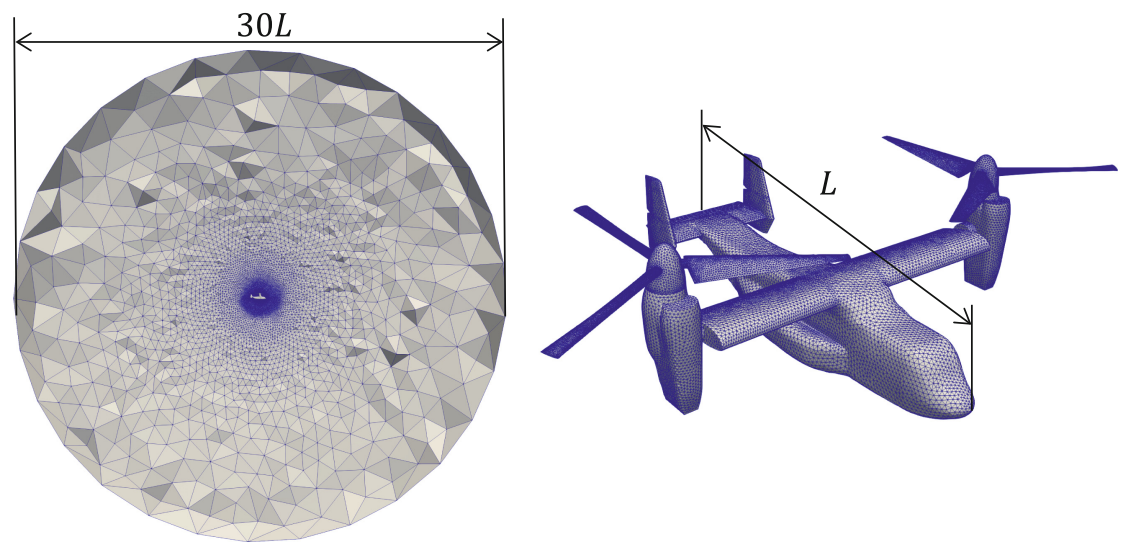

Fig. 2. Computational domain and surface grid

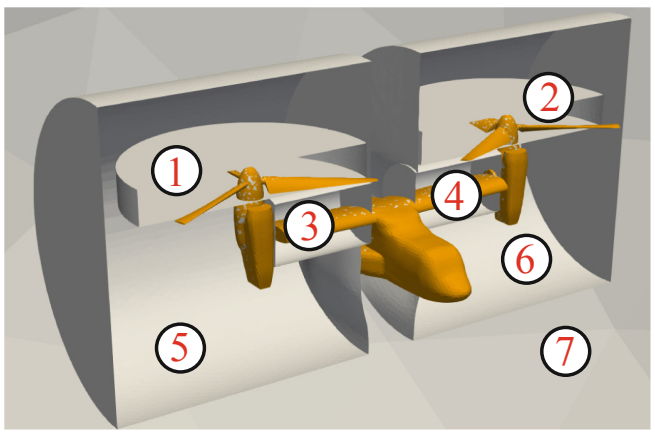

Fig. 3. Domain decomposition 


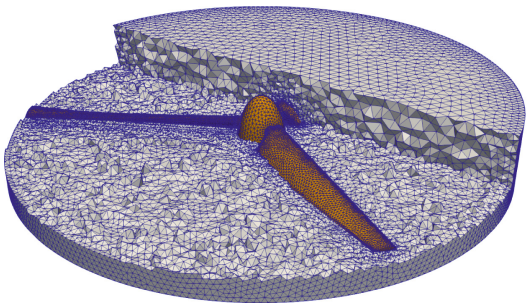

Domain 1

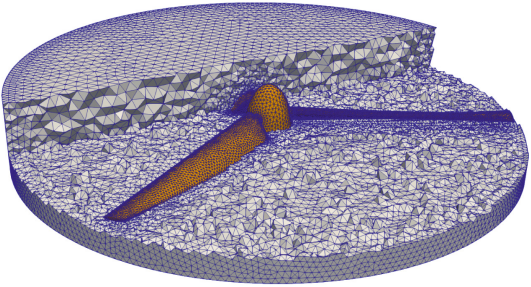

Domain2

Fig. 4. Mesh around rotors

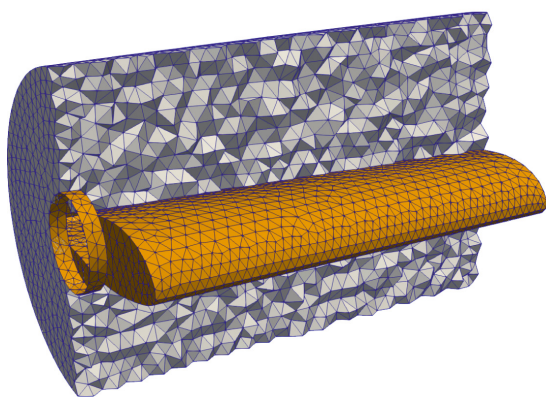

Domain3

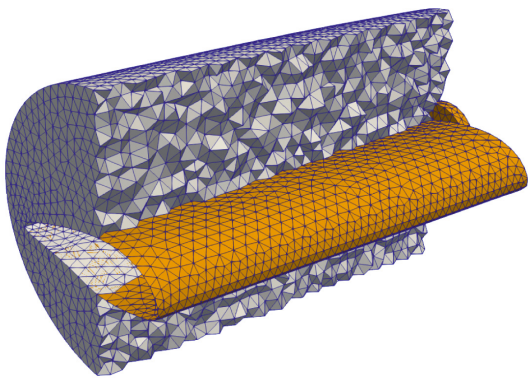

Domain4

Fig. 5. Mesh around wings

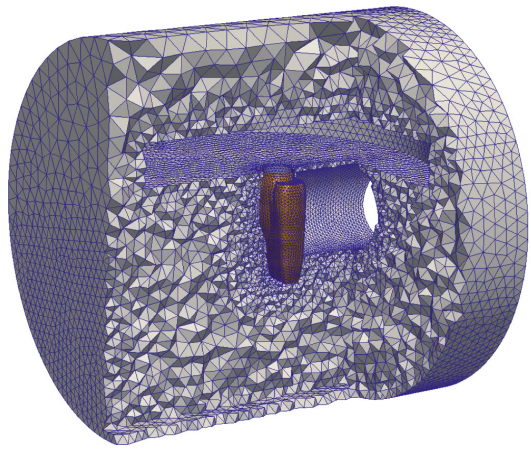

Domain5

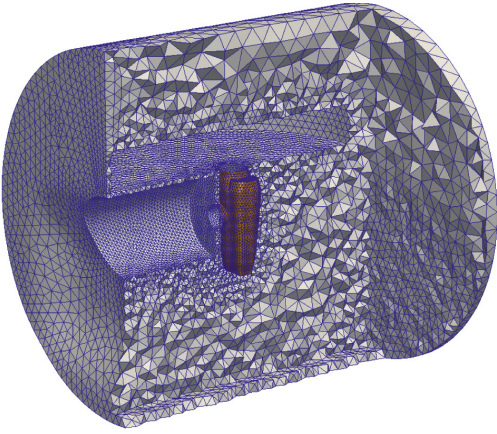

Domain6

Fig. 6. Mesh around nacelles 

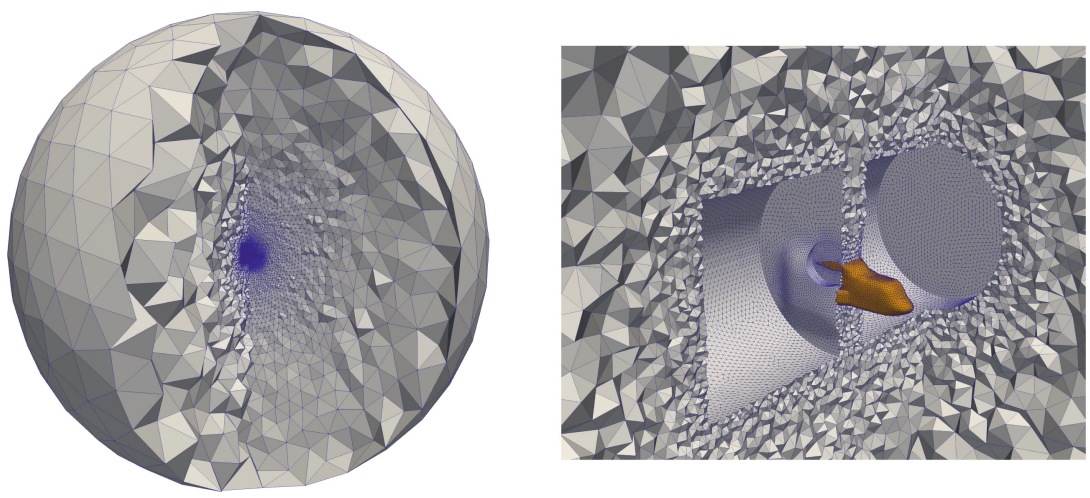

Domain7

Fig. 7. Mesh around a fuselage

\subsection{Flight Conditions}

In this simulation, a tiltrotor aircraft descends at different descent rates. The descent rates 0.02 (1340 feet/min) and 0.04 (2680 feet/min) are used for this analysis. The simulation procedure is as follows:

(1) At the start of computation, an aircraft is stationary in the air.

(2) Start to rotate two proprotors with the aircraft fixed. Then, the lift and the gravity are balanced by adjusting collective pitch.

(3) Unlock aircraft having been fixed and make an aircraft descend, adjusting collective pitch to maintain the constant descent rate.

The pitching motion of the aircraft is controlled by tilting two proprotor disks forward or backward at same time. In addition, the rolling of the aircraft is controlled by providing a difference between left and right collective pitches.

\section{Results}

\subsection{Movement of Tiltrotor Aircraft}

As a result of computation with fluid-rigid body interaction, an aircraft descended under the influence of the surrounding flow field. The trajectory of descent of an aircraft is shown in Fig. 8. The left is the result at descent rate of 0.02 and the right is the result at the descent rate of 0.04 . At descent rate is 0.02 , orientation of an aircraft slightly disturbs during the descent, but there is no significant change overall. On the other hand, at descent rate 0.04 , it is confirmed that orientation of an aircraft is largely disturbed as time elapses. Time changes of pitch angle of an aircraft about these two cases is indicated by Fig. 9. As shown in the figure, in both cases, pitch angles start to change around $t=300$. In the case of $V_{z}=0.02$, no further change is seen after orientation of an aircraft is initially disturbed. On the other hand, in the case of $V_{z}=0.04$, pitch angle of 
an aircraft gradually increases, and is expected to increase in the future. From the above, it was confirmed that orientation of an aircraft was more disturbed as the descent rate increased.
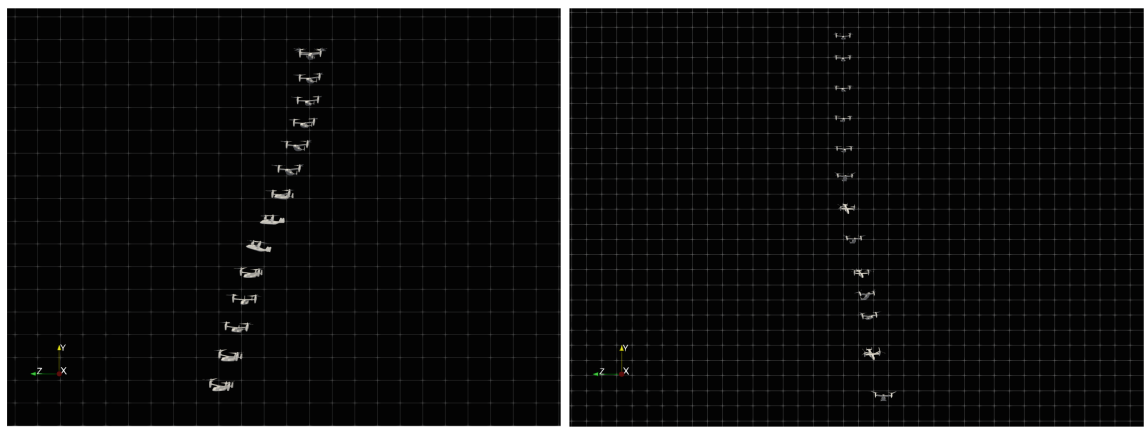

Fig. 8. Trajectory of an aircraft movement at descent rate 0.02 (left) and 0.04 (right)

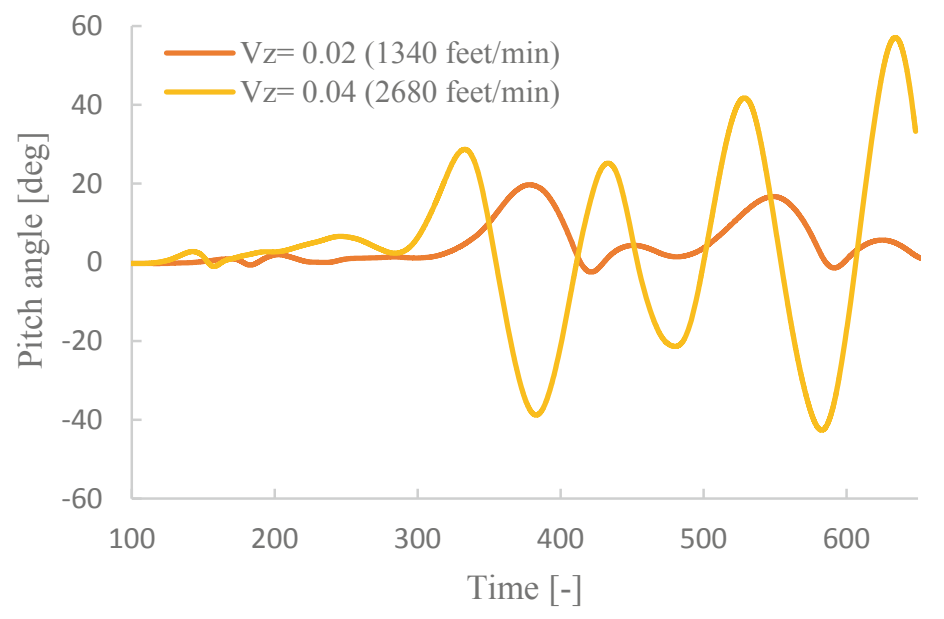

Fig. 9. Pitch angle of an aircraft at each descent rate $V_{z}$

\subsection{Flow Fields}

Figure 10 is isosurfaces $(V=0.1)$ of magnitude of the flow velocity around the tiltrotor aircraft during descent at descent rate 0.02. It illustrates a state from the start of descent. The figure at the upper left shows the state immediately after the descent started, where the blade pitch has been reduced and acceleration has started to the target descent rate. At this time, the iso-surface of the magnitude value of the velocity is in the state of clinging around the rotors. In this situation, you can see that the lift loses to gravity and the aircraft descends. The right figure next to that shows a little advanced time level. 


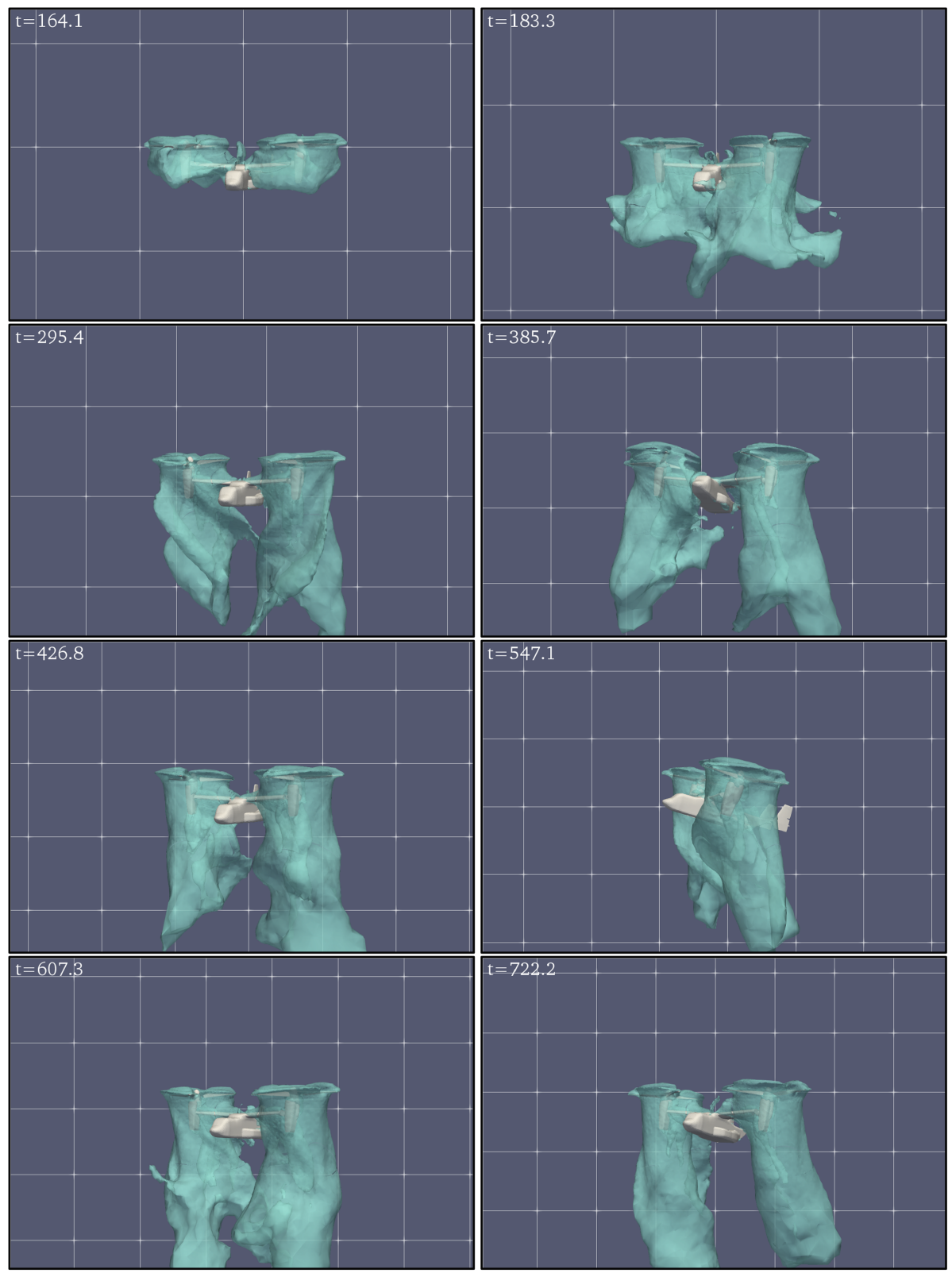

Fig. 10. Isosurfaces of magnitude of flow velocity $(V=0.1)$ at descent rate 0.02 (about 1340 feet/min) 


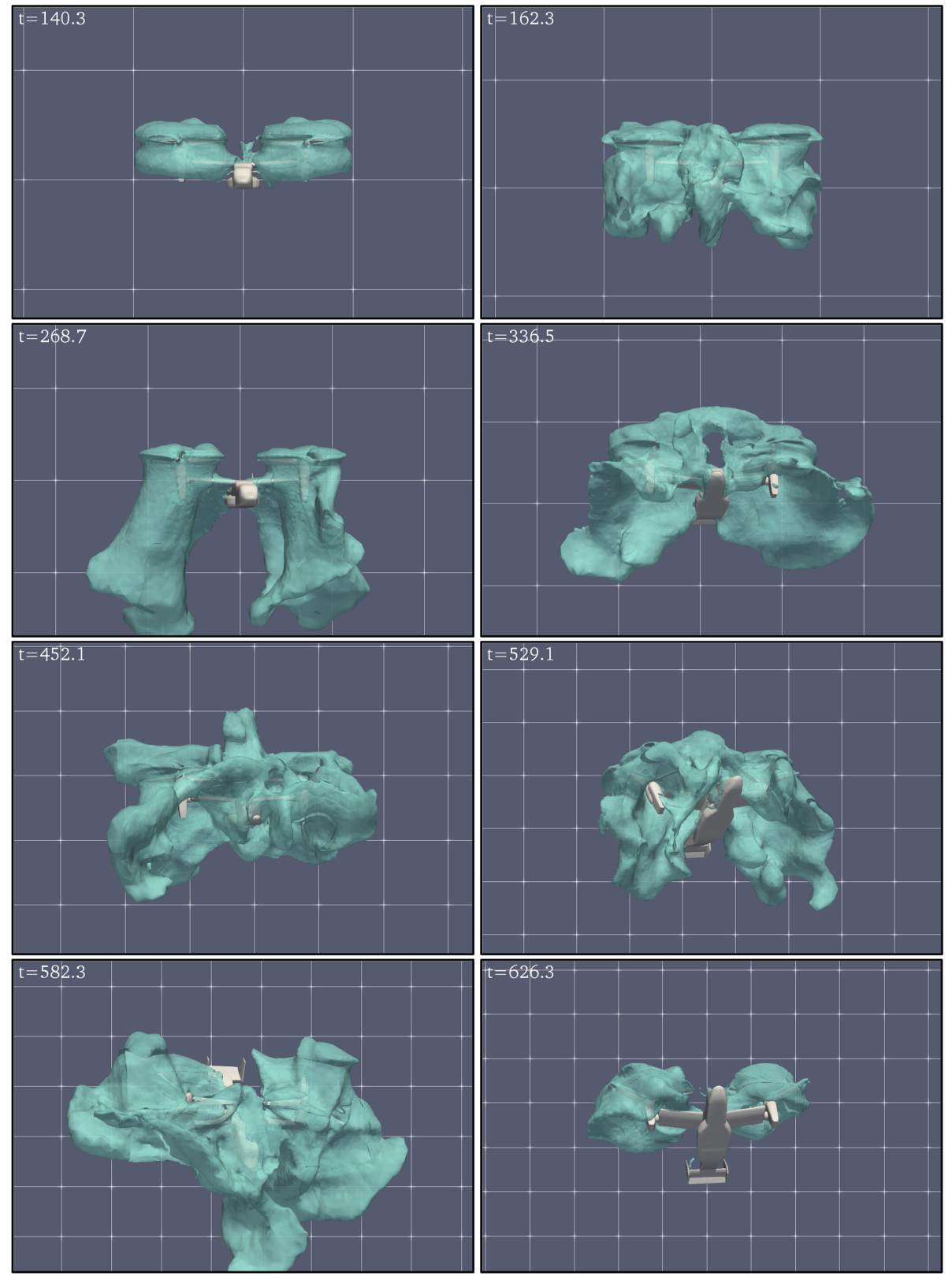

Fig. 11. Isosurfaces of magnitude of flow velocity $(V=0.1)$ at descent rate 0.04 (about 2680 feet/min) 
As the velocity of the aircraft approaches the target descent rate, the blade pitch is adjusted so that lift and gravity balance and maintain the constant speed of the body. As a result, the downwash blowing down from the rotors appeared again. Therefore, at this point, it can be considered that the aircraft obtained the lift as a reaction force against the downwash. After that, the body descends at an almost constant speed. During the descent, it is constantly confirmed that downwash is blown down from left and right proprotors. This indicates that the aircraft flies with proper lift. Next, Fig. 11 is isosurfees $(V=0.1)$ of magnitude of the flow velocity around an aircraft during descent at descent rate 0.04 , illustrating a state from the start of descent. As in the previous case, the upper left figure shows the state just after the aircraft has started to descent. The isosurface has its state clinging around the rotors due to the acceleration to the target descent rate. Then, for a while after reaching the target descent rate, you can see the downwash that is blown down from the left and right rotors (about $t=270$ ). However, unlike previous case, an aircraft begins to pitch and the surrounding airflow begins to be greatly disturbed after a while. At this point, the downwash which has been blown down from left and right proprotors disappeared. Without the downwash, the proprotors is not able to get enough lift. Moreover, once this turbulence has occurred, vicious circle between large changes in the attitude and turbulence of the fluid around the aircraft continue to repeat, increasing the instability of the body. As a result, the aircraft loses control to maintain its flight attitude.

Figure 12 and Fig. 13 show visualization of flow velocity vectors in a typical state during descent at each descent rate. At descent rate 0.02 , it is confirmed that air flows downward from proprotors. On the other hand, at descent rate 0.04 , it is confirmed that flow velocity vector is swirling at tip of proprotor. This is a vortex ring state, also known as power settling. In this situation, airflows that should be blown down from rotors is immediately recirculated to the rotors and results in a decrease in lift provided by that. Therefore, it is considered that an orientation control by proprotors does not work sufficiently and the aircraft becomes more unstable.

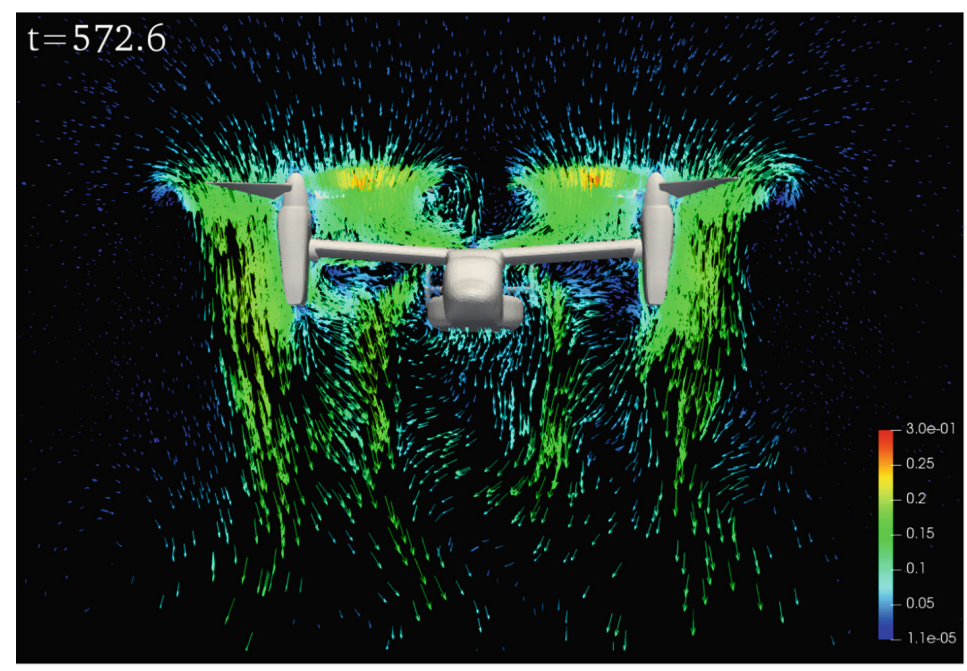

Fig. 12. Visualization of flow velocity vectors at descent rate 0.02 


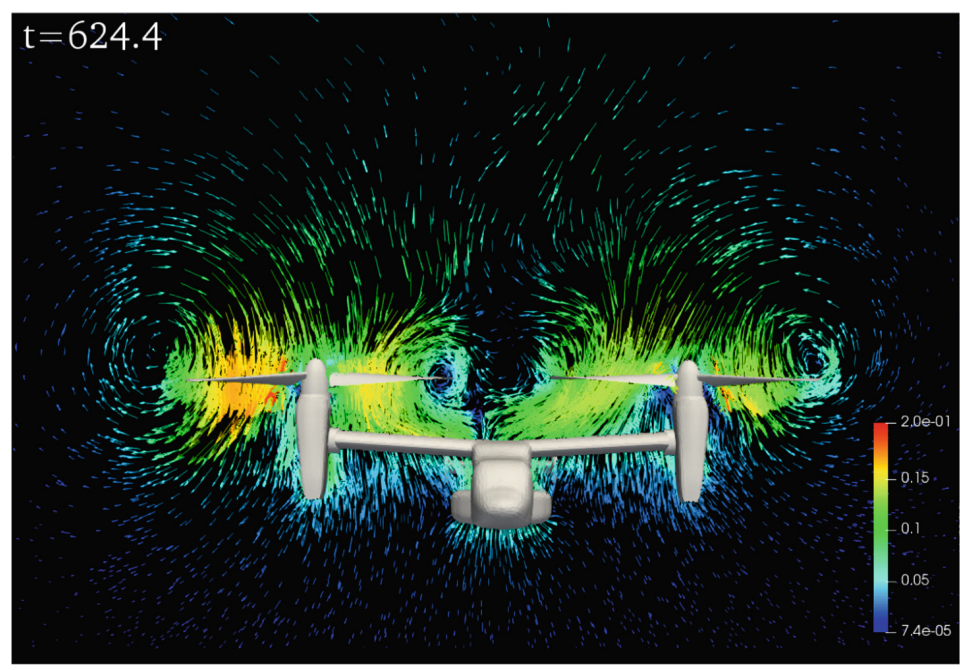

Fig. 13. Visualization of flow velocity vectors at descent rate 0.04

\section{Conclusions}

Six degrees of freedom descending flight simulation was conducted for a tiltrotor aircraft represented by V-22 Osprey, considering interaction of fluid and rigid body. To solve complicated moving boundary problem, a combination of the MCD method based on the unstructured moving-grid finite-volume method and the multi-axis sliding mesh method was adopted as numerical approach. Descending simulation is computed in two cases: descent rate 0.02 and 0.04 . The results of movement of descending aircraft was presented by trajectory and pitch angle. Next, the state of flow fields around the aircraft was presented in a form of visualization of an isosurface of flow velocity and flow velocity vectors. At descent rate 0.02 , the aircraft continued a stable descent flight throughout. At descent rate 0.04, it is confirmed that orientation of an aircraft is largely disturbed as time elapses. At that time, it was found from the flow velocity vectors that the aircraft has fallen into vortex ring state. This flight simulation qualitatively showed that a tiltrotor aircraft would become unstable and fall into vortex ring state during flight at high descent rate.

Acknowledgements. This publication was subsidized by JKA through its promotion funds from KEIRIN RACE.

\section{References}

1. Gertler, J.J.: V-22 osprey tilt-rotor aircraft: background and issues for congress. In: Library of Congress Washington DC Congressional Research Service, p. 57 (2009)

2. Johnson, W.: Model for Vortex Ring State Influence on Rotorcraft Flight Dynamics. NASA Ames Research Center, P76, Moffett Field, CA, United States (2005) 
3. Betzina, M.D.: Tiltrotor descent aerodynamics: a small-scale experimental investigation of vortex ring state. In: American Helicopter Society 57th Annual Forum, Washington, D.C. (2001)

4. Brown, R.E., Leishman, J.G., Newman, S.J., Perry, FJ.: Blade twist effects on rotor behaviour in the vortex ring state. In: 28th European rotorcraft forum, Bristol (2002)

5. Grzegorczyk, K.: Analysis of influence of helicopter descent velocity changes on the phenomena of vortex ring state. Adv. Sci. Technol. Res. J. 7(17), 35-41 (2013)

6. Surmacz, K., Ruchała, P., Stryczniewicz, W.: Wind tunnel tests of the development and demise of Vortex Ring State of the rotor. In: Advances in Mechanics: Theoretical, Computational and Interdisciplinary Issues, Proceedings of the 3rd Polish Congress of Mechanics (PCM) and 21st International Conference on Computer Methods in Mechanics (CMM), Gdansk, 8-11 September 2015, pp. 551-554. CRC Press, Leiden (2016)

7. Yamakawa, M., Matusno, K.: Unstructured moving-grid finite-volume method for unsteady shocked flows. J. Comput. Fluids Eng. 10(1), 24-30 (2005)

8. Obayashi, S.: Freestream capturing for moving coordinates in three dimensions. AIAA J. 30, 1125-1128 (1992)

9. Yamakawa, M., Chikaguchi, S., Asao, S.: Numerical simulation of tilt-rotor plane using multi axes sliding mesh approach. In: The 27th International Symposium on Transport Phenomena, Honolulu (2016)

10. Watanabe, K., Matsuno, K.: Moving computational domain method and its application to flow around a high-speed car passing through a hairpin curve. J. Comput. Sci. Technol. 3(2), 449-459 (2009)

11. Yamakawa, M., Mitsunari, N., Asao, S.: Numerical simulation of rotation of intermeshing rotors using added and eliminated mesh method. Procedia Comput. Sci. 108, 1883-1892 (2017)

12. Asao, S., et al.: Simulations of a falling sphere with concentration in an infinite long pipe using a new moving mesh system. Appl. Thermal Eng. 72, 29-33 (2014)

13. Asao, S., et al.: Parallel computations of incompressible flow around falling spheres in a long pipe using moving computational domain method. Comput. Fluids 88, 850-856 (2013)

14. Yamakawa, M., Takekawa, D., Matsuno, K., Asao, S.: Numerical simulation for a flow around body ejection using an axisymmetric unstructured moving grid method. Comput. Thermal Sci. 4(3), 217-223 (2012)

15. Takii, A., Yamakawa, M., Asao, S., Tajiri, K.: Six degrees of freedom numerical simulation of tilt-rotor plane. In: Rodrigues, J.M.F., et al. (eds.) ICCS 2019. LNCS, vol. 11536, pp. 506-519. Springer, Cham (2019). https://doi.org/10.1007/978-3-030-22734-0_37

16. Roe, P.L.: Approximate riemann solvers parameter vectors and difference schemes. J. Comput. Phys. 43, 357-372 (1981)

17. Hishida, M., Hashimoto, A., Murakami, K., Aoyama, T.: A new slope limiter for fast unstructured CFD solver FaSTAR. In: Proceedings of 42nd Fluid Dynamics Conference/Aerospace Numerical Simulation Symposium, pp. 1-10 (2010). (in Japanese)

18. Ito, Y., Nakahashi, K.: Surface triangulation for polygonal models based on CAD data. Int. J. Numer. Methods Fluids 39(1), 75-96 (2002)

19. Ito, Y.: Challenges in unstructured mesh generation for practical and efficient computational fluid dynamics simulations. Comput. Fluids 85, 47-52 (2013) 